\title{
XLVII. On the constitution of the luminiferous æther
}

\section{G.G. Stokes M.A.}

To cite this article: G.G. Stokes M.A. (1848) XLVII. On the constitution of the luminiferous æther , Philosophical Magazine, 32:216, 343-349, DOI: 10.1080/14786444808645996

To link to this article: http://dx.doi.org/10.1080/14786444808645996

曲 Published online: 30 Apr 2009.

Submit your article to this journal ๔

Џ Article views: 7

Q View related articles $₫$ 
This series is precisely that which Professor Challis has found on page 281. And thus it appears that the solution in which that series is concerned does in reality express the interference of an indefinite number of plane waves, all travelling with the velocity assigned by the ordinary theory, and all indefinitely extended in the direction of their planes. These waves cannot all originate from one source, and therefore this solution does not relate to the problem of sound, in its ordinary acceptation.

I will only further allude to the difficulty to which Professor Challis adverts in his last paragraph. The difficulty must arise, in some way, from misunderstanding on words. The most trifling exanination of the process in the investigation of the velocity of sound serves to show that the velocity does not depend on the absolute pressure of the air in its normal state of density, but upon the proportion of the change of pressure to the change of density. This is increased by the suddenness of condensation in one part, which when the elastic force is great makes it still greater, and by the suddenness of rarefaction in another part, which when the elastic force is small makes it still smaller; thus in both ways increasing the change of pressure.

I am, Gentlemen,
Your obedient Servant,
$\begin{aligned} & \text { Ruyal Observatory, Greenwich, } \\ & \text { April 10, 1848. }\end{aligned}$
G. B. Arry.

XLVII. On the Constitution of the Luminiferous AEther. By G. G. Stokes, M.A., Fellow of Pembroke College, Cambridge*.

THE phænomenon of aberration may be reconciled with 1 the undulatory theory of light, as I have already shown (Phil. Mag., vol. xxvii. p. 9), without making the violent supposition that the rether passes freely through the earth in its motion round the sun, but supposing, on the contrary, that the sether close to the surface of the earth is at rest relatively to the earth. This explanation requires us to suppose the motion of the ather to be such, that the expression usually denoted by $u d x+v d y+r o d z$ is an exact differential. It becomes an interesting question to inquire on what physical properties of the ather this sort of motion can be explained. Is it sufficient to consider the zther as an ordinary fluid, or must we have recourse to some property which does not exist in ordinary fluids, or, to speak more correctly, the existence of which

* Communicated by the Author. 
has not been made manifest in such fluids by any phænomenon hitherto observed? I have already attempted to offer an explanation on the latter supposition (Phil. Mag., vol xxix. p. 6). Professor Challis, in his last communication, has considered the æther as an ordinary fluid.

In my paper last referred to, I have expressed my belief that the motion for which $u d x+\& c$. is an exact differential, which would take place if the æther were like an ordinary fluid, would be unstable; I now propose to prove the same mathematically, though by an indirect method.

Even if we supposed light to arise from vibrations of the æether accompanied by condensations and rarefactions, analogous to the vibrations of the air in the case of sound, since such vibrations would be propagated with about 10,000 times the velocity of the earth, we might without sensible error neglect the condensation of the rether in the motion which we are considering. As far as the case in hand is concerned, Professor Challis might have regarded $p$ as constant, and treated $p$ as he has treated $s$. Suppose, then, a sphere to be moving uniformly in a homogeneous incompressible fluid, the motion being such that the square of the velocity may be neglected. There are many obvious phænomena which clearly point out the existence of a tangential force in fluids in motion, analogous in many respects to friction in the case of solids. When this force is taken into account, the equations of motion become (Cambridge Philosophical Transactions, vol.viii.p. 297)

$$
\frac{d p}{d x}=-\rho \frac{d u}{d t}+\mu\left(\frac{d^{2} u}{d x^{2}}+\frac{d^{2} u}{d y^{2}}+\frac{d^{2} u}{d z^{2}}\right), \quad \text {. . }
$$

with similar equations for $y$ and $z$. In these equations the square of the velocity is omitted, according to the supposition made above, $\rho$ is considered constant, and the fluid is supposed not to be acted on by external forces. We have also the equation of coninuity

$$
\frac{d u}{d x}+\frac{d v}{d y}+\frac{d w}{d z}=0, . . . . .
$$

and the conditions, (1) that the fluid at the surface of the sphere shall be at rest relatively to the surface, (2) that the velocity shall vanish at an infinite distance.

For my present purpose it is not requisite that the equations such as (1.) should be known to be true experimentally; if they were even known to be false they would be sufficient, for they may be conceived to be true without mathematical absurdity. My argument is this. If the motion for which $u d x+\ldots$ is an exact differential, which would be obtained 
from the common equations, were stable, the motion which would be obtained from equations (1.) would approach inde. finitely, as $\mu$ vanished, to one for which $u d x+\ldots$ was an exact differential, and therefore, for anything proverl to the contrary, the latter motion might be stable; but if, on the contrary, the motion obtained from (1.) should turn out totally different from one for which $u d x+\ldots$ is an exact differential, the latter kind of motion must necessarily be unstable.

Conceive a velocity equal and opposite to that of the sphere impressed both on the sphere and on the fluid. It is easy to prove that $u d x+\ldots$ will or will not be an exact differential after the velocity is impressed, according as it was or was not such before. 'The sphere is thus reduced to rest, and the problem becomes one of steady motion. The solution which I an about to give is extracted from some researches in which I am engaged, but which are not at present published. It would occupy far too much room in this Magazine to enter into the mode of obtaining the solution : but this is not necessary; for it will probably be allowed that there is but one solution of the equations in the case proposed, as indeed readily follows from physical considerations, so that it will be sufficient to give the result, which may be verified by differentiation.

Let the centre of the sphere be taken for origin; let the direction of the real motion of the sphere make with the axes angles whose cosines are $l, m, n$, and let $\nu$ be the real velocity of the sphere; so that when the problem is reduced to one of steady motion, the fluid at a distance from the sphere is moving in the opposite direction with a velocity $v$. Let $a$ be the sphere's radius : then we have to satisfy the general equations (1.) and (2.) with the particular conditions

$$
\begin{aligned}
& u=0, \quad v=0, \quad w=0, \text { when } r=a ; . . . \\
& u=-l \nu, \quad v=-m \nu, \quad w=-n v, \text { when } r=\infty,
\end{aligned}
$$

$r$ being the distance of the point considered from the centre of the sphere. It will be found that all the equations are satisfied by the following values,

$$
\begin{gathered}
p=\Pi+\frac{3}{2} \mu \nu \frac{a}{r^{3}}(l x+m y+n z), \\
u=\frac{3}{4} \nu\left(\frac{a}{r^{3}}-\frac{a^{3}}{r^{5}}\right) x(l x+m y+n z)+l \nu\left(\frac{1}{4} \frac{a^{3}}{r^{3}}+\frac{3}{4} \frac{a}{r}-1\right),
\end{gathered}
$$

with symmetrical expressions for $v$ and $w$. II is here an arbitrary constant, which evidently expresses the value of $p$ at an infinite distance. Now the motion defined by the above ex- 
pressions does not tend, as $\mu$ vanishes, to become one for which $u d x+\ldots$ is an exact differential, and therefore the motion which would be obtained by supposing $u d x+\ldots$ an exact differential, and applying to the æther the common equations of hydrodynamics, would be unstable. The proof supposes the motion in question to be steady; but such it may be proved to be, if the velocity of the earth be regarded as uniform, and an equal and opposite velocity be conceived impressed both on the earth and on the ather. Hence the stars would appear to be displaced in a manner different from that expressed by the well-known law of aberration.

When, however, we take account of a tangential force in the æther, depending, not on relative velocities, or at least not on relative velocities only, but on relative displacements, it then becomes possible, as I have shown (Phil. Mag., vol. xxix. p. 6), to explain not only the perfect regularity of the motion, but also the circumstance that $u d x+\ldots$ is an exact differential, at least for the æther which occupies free space; for as regards the motion of the ather which penetrates the air, whether about the limits of the atmosphere or elsewhere, I do not think it prudent, in the present state of our knowledge, to enter into speculation; I prefer resting in the supposition that $u d x+\ldots$ is an exact differential. According to this explanation, any nascent irregularity of motion, any nascent deviation from the motion for which $u d x+\ldots$ is an exact differential, is carried off into space, with the velocity of light, by transversal vibrations, which as such are identical in their physical nature with light, but which do not necessarily produce the sensation of light, either because they are too feeble, as they probably would be, or because their lengths of wave, if the vibrations take place in regular series, fall beyond the limits of the visible spectrum, or because they are discontinuous, and the sensation of light may require the succession of a number of similar vibrations. It is certainly curious that the astronomical phænomenon of the aberration of light should afford an argument in support of the theory of transversal vibrations.

Undoubtedly it does violence to the ideas that we should bave been likely to form $\grave{a}$ priori of the nature of the ather, to assert that it must be regarded as an elastic solid in treating of the vibrations of light. When, however, we consider the wonderful simplicity of the explanations of the phænomena of polarization when we adopt the theory of transversal vibrations, and the difficulty, which to me at least appears quite insurmountable, of explaining these phænomena by any vibrations due to the condensation and rarefaction of an elastic fluid such as air, it seems reasonable to suspend our judge- 
ment, and be content to learn from phænomena the existence of forces which we should not beforehand have expected. The explanations which I had in view are those which belong to the geometrical part of the theory; but the deduction, from dynamical calculations, of the laws which in the geometrical theory take the place of observed facts must not be overlooked, although here the evidence is of a much more complicated character.

The following illustration is advanced, not so much as explaining the real nature of the æther, as for the sake of offering a plausible mode of conceiving how the apparently opposite properties of solidity and fluidity which we must attribute to the æther may be reconciled.

Suppose a small quantity of glue dissolved in a little water, so as to form a stiff jelly. This jelly forms in fact an elastic solid : it may be constrained, and it will resist constraint, and return to its original form when the constraining force is removed, by virtue of its elasticity; but if we constrain it too far it will break. Suppose now the quantity of water in which the glue is dissolved to be doubled, trebled, and so on, till at last we have a pint or a quart of glue water. The jelly will thus become thinner and thinner, and the amount of constraining force which it can bear without being dislocaied will become less and less. At last it will become so far fluid as to mend itself again as soon as it is dislocated. Yet there seems hardly sufficient reason for supposing that at a certain stage of the dilution the tangential force whereby it resists constraint ceases all of a sudden. In order that the medium should not be dislocated, and therefore should have to be treated as an elastic solid, it is only necessary that the amount of constraint should be very small. The medium would however be what we should call a fluid, as regards the motion of solid bodies through it. The velocity of propagation of normal vibrations in our medium would be nearly the same as that of sound in water ; the velocity of propagation of transversal vibrations, depending as it does on the tangential elasticity, would become very small. Conceive now a medium having similar properties, but incomparably rarer than air, and we have a medium such as we may conceive the ather to be, a fluid as regards the motion of the earth and planets through it, an elastic solid as regards the small vibrations which constitute light. Perbaps we should get nearer to the true nature of the æther by conceiving a medium bearing the same relation to air that thin jelly or glue water bears to pure water. The sluggish transversal vibrations of our thin jelly are, in the case of the sether, replaced by vibrations propagated with a velocity of nearly 
200,000 miles in a second : we should expect, $a$ priori, the velocity of propagation of normal vibrations to be incomparably greater. This is just the conclusion to which we are led quite independently, from dynamical principles of the greatest generality, combined with the observed phænomena of optics*.

I take this opportunity of making a few remarks on my explanation of aberration (Phil. Mag., vol. xxvii. p. 9), more especially as Professor Challis's words at page 168 of the present volume would naturally lead to the idea, which, however, I believe was not intended, that I had only explained the change in the direction of the normal to a wave of light, so that something was wanting to complete, on the suppositions adopted, the explanation of aberration. To prevent misapprehension, I would observe, that in the explanation of aberration $I$ here include the explanation of the rectilinear propagation of light, if the explanation of aberration be divided into two parts ; the first, the explanation of rectilinear propagation; the second, the explanation of aberration on the assumption of rectilinear propagation. To my own mind, the undulatory theory cannot be said to explain aberration unless it explains, either rectilinear propagation, or what is equivalent to it; for had the stars never been observed, I should have thought it excessively improbable that the path of a ray was rectilinear in the neighbourhood of the earth. As to the necessity for an explanation of aberration on any theory of light, I quite agree with Professor Challis, as he has stated. Indeed, if I ever appeared to differ from him on this point, it was not because I held a different opinion, but because I failed to catch his meaning.

In my first paper on aberration, it is true that I did not investigate the nature of the path of a ray of light in space; but this was only because the method I employed did not require any such investigation. I showed that, on the suppositions adopted, the path of a ray, not in space but relatively to the earth, was in the immediate neighbourhood of the observer directed to the apparent place of the heavenly body from which it came, that is, its place as affected by the observed aberration; and that was sufficient for my purpose. My explanation was not even deficient in consequence of not taking account of the light coming from the wire to which the star was referred; for according to my method, everything was reduced to the case in which the earth and the æther in its immediate neighbourhood are supposed to be at rest; so

- See the introduction to an admirable memoir by Green, On the Reflexion and Refraction of Light.-Cambridge Philosophical Transactions, vol, vii. p. 1. 
that there was no more occasion to notice explicitly the light coming from the wires, than there would have been if the earth had really been at rest. While, however, I would vindicate my explanation from any flaw or deficiency of reasoning (unless the not noticing formally and explicitly the light coming from the wires be regarded as such), I allow that, without investigation, I fancied the path of a ray in space to be curvilinear. It was first virtually proved by Professor Challis, though not explicitly stated, that the path was rectilinear throughout. Consequently the angle $p e q$ (Phil. Mag., vol.xxvii. p. 14), which $I$ argued was insensible, is in fact zero. The method which consists in considering the rectilinear propagation of light as resulting from the supposition that $u d x+\ldots$ is an exact differential, and then the law of aberration as resulting from the rectilinear propagation, instead of considering the whole at once, has the advantage of showing that we are at liberty to suppose the velocity of the ather at the surface of the earth to be of any amount relatively to the surface. I had not contemplated this case; for it was the precise object of my investigation to get rid of the apparent necessity of supposing the æether to be rushing through the air and through the earth itself as the earth moves round the sun.

XLVIII. On Shooting Stars. By J. P. Joule, Corresponding Member of the Royal Academy of Sciences, Turin, Sccretary to the Literary and Philosophical Society, Manchester*.

T HAVE read with much interest the valuable papers on 1 shooting stars inserted by Sir J. W. Lubbock in the Numbers of the Philosophical Magazine for February and March. This philosopher seems to have placed the subject in a fair way for satisfactory solution. He has advanced three hypotheses to account for the sudden disappearance of these bodies, the last of which he has enabled us to prove or disprove by actual observation.

I have for a long time entertained an hypothesis with respect to shooting stars, similar to that advocated by Chladni to account for meteoric stones, and have reckoned the ignition of these miniature planetary bodies by their violent collision with our atmosphere, to be a remarkable illustration of the doctrine of the equivalency of heat to mechanical power or vis viva. In a popular lecture delivered in Manchester on the 28th of April 1847, I said, "You have, no doubt, frequently observed what are called shooting stars, as they appear to emerge from the

* Communicated by the Author. 